\title{
Bidirectional Regulation of COX-2 Expression Between Cancer Cells and Macrophages
}

\author{
MARIA ISABEL CARVALHO ${ }^{1,2,3}$, RODOLFO BIANCHINI ${ }^{3}$, JUDIT FAZEKAS-SINGER ${ }^{3,4}$, \\ INA HERRMANN ${ }^{5}$, IRENE FLICKINGER ${ }^{6}$, JOHANN G. THALHAMMER ${ }^{5}$, ISABEL PIRES ${ }^{1,2}$, \\ ERIKA JENSEN-JAROLIM ${ }^{3,4}$ and FELISBINA L. QUEIROGA ${ }^{2,7,8}$ \\ ${ }^{1}$ Animal and Veterinary Research Centre, ${ }^{2}$ Department of Veterinary Sciences, and \\ ${ }^{8}$ Center for Research and Technology of Agro-Environment and Biological Sciences, \\ University of Trás-os-Montes and Alto Douro, Vila Real, Portugal; \\ ${ }^{3}$ The Interuniversity Messerli Research Institute of the University of Veterinary Medicine Vienna, \\ Medical University Vienna and University Vienna, Vienna, Austria; \\ ${ }^{4}$ Institute of Pathophysiology and Allergy Research, Center of Pathophysiology, \\ Infectiology and Immunology, Medical University Vienna, Vienna, Austria; \\ ${ }^{5}$ Division of Dermatology, Internal Medicine Small Animals, University Clinics of Horses and Small Animals, \\ University of Veterinary Medicine, Vienna, Austria; \\ ${ }^{6}$ Division of Oncology, Small Animal Internal Medicine, University Clinics of Horses and Small Animals, \\ University of Veterinary Medicine, Vienna, Austria; \\ ${ }^{7}$ Center for the Study of Animal Sciences, Institute of Sciences, \\ Technologies and Agro-environment, University of Porto, Porto, Portugal
}

\begin{abstract}
Background/Aim: Our aim was to investigate the crosstalk between tumor and immune cells (M2 macrophages) and its effects on cyclo-oxygenase-2 (COX2) regulation in canine mammary tumors $(C M T)$. Materials and Methods: Sh1b CMT cells and human BT474 mammary or HT29 colon cancer cells were co-cultured with canine peripheral blood mononuclear cells (PBMCs) or with macrophage-like differentiated THP1 monocytes (dTHP1). Intracellular COX2 expression by PBMCs, dTHP1 and cancer cells was evaluated by flow cytometry. Results: Coculturing of Shlb and canine PBMCs induced COX2 overexpression in CMT cells. In turn, COX2 expression by PBMCs, mostly CD68 ${ }^{+}$macrophages, was attenuated by coculture with Shlb ( $p=0.0001)$. In accordance, co-culture with dTHP1 prompted intracellular production of COX2 in both Sh1b CMT cells and HT29 human colon cancer cells and reduced production of COX2 in BT474 human mammary cancer cells. The intracellular COX2 expression from dTHP1
\end{abstract}

Correspondence to: Professor Felisbina Queiroga, Department of Veterinary Sciences, University of Trás-os-Montes and Alto Douro, Quinta dos Prados, 5000-801 Vila Real, Portugal. Tel: +351 917826982, Fax: +351 259350480, e-mail fqueirog@utad.pt

Key Words: Canine mammary cancer, COX-2, macrophages, Sh1b, BT474, HT29. decreased when treated with conditioned medium from cultured Shlb and HT29 cancer cells. Conclusion: Bidirectional COX2 regulation between cancer and monocytes/macrophages might shape a tolerogenic tumor microenvironment in CMT.

Tumor progression is critically dependent on the microenvironment in the surrounding stroma. The microenvironment contains a cocktail of inflammatory, regenerative and immune cells, along with all cytokines and mediators they release that may support or suppress tumor growth (1).

T-Lymphocytes and macrophages are major constituents of the inflammatory infiltrate observed in several human and canine tumors. In both species, these immune cells in mammary tumor sites produce a variety of inflammatory mediators that influence tumor progression, invasion and metastasis (2-6). Interestingly, animal and human studies report that cyclo-oxygenase-2 (COX2) overexpression in mammary tumors in particular strongly supports the development of hallmarks of cancer and disease progression (7-12).

In parallel, in human and canine mammary tumors (CMT), the presence of high numbers of intratumoral T-lymphocytes as well as of macrophages with a typical M2 phenotype is generally associated with poor overall survival (OS) $(4,13$, 14). Immunosuppression associated with T-lymphocyte infiltration has been explained by the compartment of $\mathrm{CD} 4{ }^{+} \mathrm{CD} 25^{+}$forkhead box $\mathrm{P} 3(\mathrm{FOXP} 3)^{+}$regulatory T-cells 
(Tregs) that inhibit cytotoxic activity (15-18). In contrast, the immunosuppressive and, therefore, pro-tumorous action of macrophages is much less defined.

In human breast cancer, $\mathrm{COX} 2$-derived prostaglandin $\mathrm{E}_{2}$ $\left(\mathrm{PGE}_{2}\right)$ has the ability to influence local immune responses in the tumor stroma, contributing to tumor evasion of immune surveillance $(11,19)$. A substantial body of work describes an immunosuppressive role for COX2. The $\mathrm{COX} 2 / \mathrm{PGE}_{2}$ signaling pathways may contribute to modulation of macrophages, inducing the promotion of M2-macrophages cytokines which nourish T-helper (Th) 2 immune responses $(20,21)$. The immune cell subtypes modulated by COX2 share common cytokine mediators and, more importantly, each cell subtype can also influence COX2 production, providing an autocrine mechanism for prolonging and enhancing their own immunosuppressive phenotype (22).

Collectively, these findings show that in cancer immune cells share common signaling pathways of COX2 regulation. This is relevant for mammary carcinogenesis associated with changes in immune cell profiles and functionality, possibly allowing neoplastic cells to evade attack from the immune system (22).

In contrast, in CMT only few studies have yet concentrated on the COX2 crosstalk between cancer and immune cells (23, 24). We, therefore, focused on the interplay of macrophages and tumor cells aiming to contribute to the understanding of the potential bidirectional crosstalk between them and their role in regulation of $\mathrm{COX} 2$ expression.

\section{Materials and Methods}

Cell culture. Canine mammary carcinoma cell line Sh1b was a kind gift of Dr. Gerard Rutteman (Department of Clinic Science and Companion Animals, University of Utrecht, the Netherlands) to the University of Veterinary Medicine Vienna. HT29 human colon cancer cell line, BT474 human breast carcinoma cell line and THP1 human monocytic cell line were purchased from American Type Culture Collection (Rockville, MD, USA). Sh1b was maintained in Dulbecco's modified Eagle's medium (DMEM) F12 (Gibco, Life Technologies, Carlsbad, CA, USA) supplemented with heat inactivated $10 \%$ fetal calf serum (FCS), $2 \mathrm{mM} \mathrm{L}$-glutamine and $10 \mu \mathrm{g} / \mathrm{ml}$ gentamicin sulfate. BT474 was maintained in DMEM (Gibco) with $10 \%$ FCS, 2 mM L-glutamine, penicillin $(100 \mathrm{U} / \mathrm{ml})$ and streptomycin $(100 \mu \mathrm{g} / \mathrm{ml})$. HT29 and THP1 were cultured in Roswell Park Memorial Institute (RPMI)-1640 medium (Gibco) supplemented with 10\% FCS, 2 mM L-glutamine, penicillin $(100 \mathrm{U} / \mathrm{ml})$ and streptomycin $(100 \mu \mathrm{g} / \mathrm{ml})$. Cells were incubated at $37^{\circ} \mathrm{C}$ in a humidified atmosphere containing $95 \%$ air and $5 \% \mathrm{CO}_{2}$.

Collection of tumor cell line culture supernatants. Sh1b, HT29 and BT474 cells were grown in flasks at $80-90 \%$ confluence and harvested with trypsin. After that, $8 \times 105$ cells $/ \mathrm{ml}$ were seeded into 6-well culture plate containing $2 \mathrm{ml}$ per well of DMEM F12 for Sh1b, DMEM for BT474 or RPMI-1640 for HT29 cells. Cells were incubated at $37^{\circ} \mathrm{C}$ in a humidified atmosphere containing $95 \%$ air and $5 \% \mathrm{CO}_{2}$. After $24 \mathrm{~h}$ incubation, the medium of all cell lines was changed for fresh RPMI-1640 and the culture supernatants were collected $24 \mathrm{~h}$ later and stored at $-20^{\circ} \mathrm{C}$ until needed for the corresponding experiments.

THP1 differentiation in response to phorbol myristate acetate (PMA) and treatment with culture supernatant. THP1 cells $\left(2 \times 10^{5}\right.$ cells $/ \mathrm{ml}$ ) were seeded into a 24 -well plate containing $500 \mu \mathrm{l}$ of RPMI-1640 culture medium with $200 \mathrm{nM}$ of PMA for $72 \mathrm{~h}$. After incubation, nonattached cells were removed by aspiration. The adherent THP1 cells were washed three times with Hank's Balanced Salt Solution (HBSS) to completely remove PMA and then treated with $50 \%$ v/v of Sh1b or HT29 culture supernatant for $48 \mathrm{~h}$. CD68 and intracellular COX2 expression in macrophage-like differentiated THP1 monocytes (dTHP1) was then evaluated by flow cytometry.

Isolation of canine peripheral blood mononuclear cells (PBMCs). Blood samples from male and female spayed dogs $(n=11)$ undergoing cancer treatment in the Oncology Clinic of the University of Veterinary Medicine Vienna were used in this study. All dogs received doxorubicin-derived chemotherapy and at blood collection the treatment duration was less than 3 months. Two milliliters of blood was drawn from the cephalic vein of each dog by venipuncture into EDTA tubes. PBMC isolation was performed with the standard Ficoll-Paque solution (Sigma Chemical, St. Louis, MO, USA) density gradient centrifugation. After collecting the contents of the milky PBMC layer, cells were washed in HBSS, resuspended in DMEM, counted and incubated in RPMI-1640 culture medium until needed for the co-culture experiments.

Co-culture experimental conditions. Sh1b, HT29 and BT474 cell lines $\left(5 \times 10^{5}\right.$ cells per well in co-culture) were plated into a 24 -well plate in respective culture medium. On day 2 , the culture medium of Sh1b, HT29 and BT474 was changed for fresh RPMI-1640 supplemented with $10 \%$ FCS, 2 mM glutamine and penicillin/streptomycin antibiotic. Canine PBMCs $\left(1 \times 10^{6}\right.$ cells $\left./ \mathrm{ml}\right)$ or PMA dTHP1 $\left(2 \times 10^{5}\right.$ cells $\left./ \mathrm{ml}\right)$ were plated directly onto $24-w e l l$ plate transwell inserts $(0.4 \mu \mathrm{m}$ pore size; Corning, Life Sciences, Tewksbury, MA, USA) in RPMI-1640 culture medium and coincubated with canine mammary cancer cell line Sh1b for $48 \mathrm{~h}$. PMA dTHP1 macrophages $\left(2 \times 10^{5}\right.$ cells $\left./ \mathrm{ml}\right)$ were also co-incubated with HT29 and BT474 human cells.

Assessment of CD68, CD3 and intracellular COX2 by flow cytometry. CD68, CD3 and intracellular COX2 expression in canine PBMCs; intracellular COX2 expression in Sh1b, HT29 and BT474 cells; and CD68 and intracellular COX2 expression in dTHP1 cells was evaluated on a FACSCanto II flow cytometer (BD Biosciences, Franklin Lakes, NJ, USA).

Briefly, all cells were detached, washed three times with HBSS, and re-suspended in FACS buffer. After that, the primary antibody master mix for CD68 (clone eBioY1/82A, R-phycoerythrin-cyanine dye, $2 \mu 1$ per tube; eBioscience, San Diego, CA, USA) and CD3 (clone CA17.2A12, purified, $1 \mu$ per tube; Bio-Rad, Hercules, CA, USA) was added and cells were incubated for $30 \mathrm{~min}$ at $4^{\circ} \mathrm{C}$ in the dark. Subsequently, cells were washed with FACS buffer and incubated for $30 \mathrm{~min}$ at $4^{\circ} \mathrm{C}$ in the dark with rat anti-mouse IgG1 secondary antibody for CD3 (clone M1-14012, allophycocyanin, $1 \mu 1$ per tube; eBioscience). After washing, cells were fixed with fixation buffer (eBioscience) and permeabilized with permeabilization buffer (eBioscience) according to the manufacturer's instructions and 
A

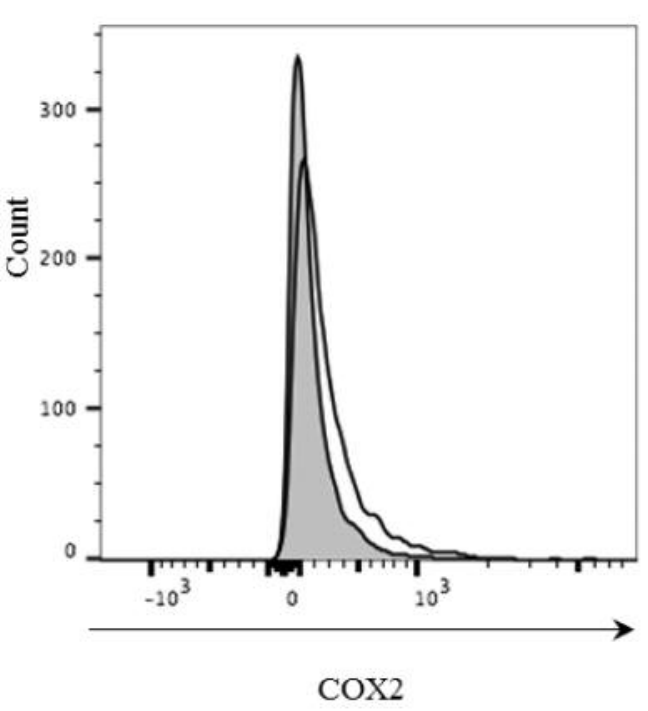

$\mathrm{C}$
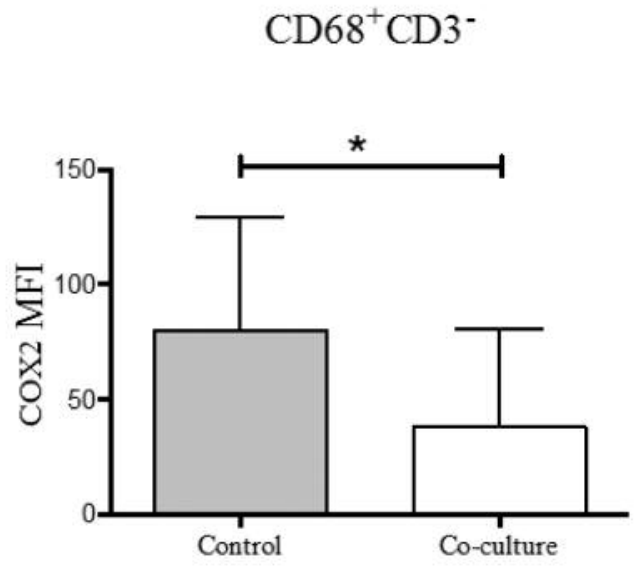

B

\section{Total PBMCs}

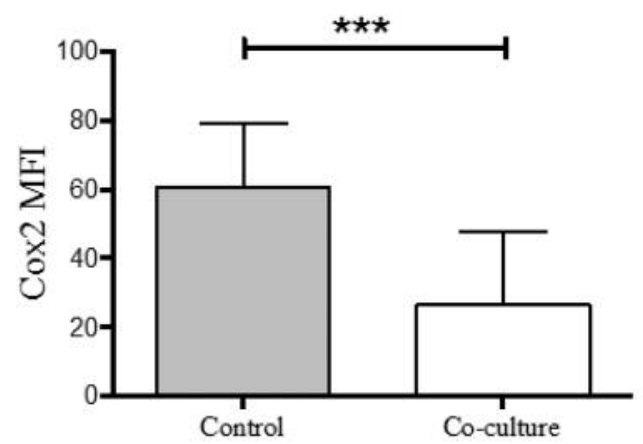

$\mathrm{D}$

\section{$\mathrm{CD}^{-{ }^{-} \mathrm{CD} 3^{+}}$}

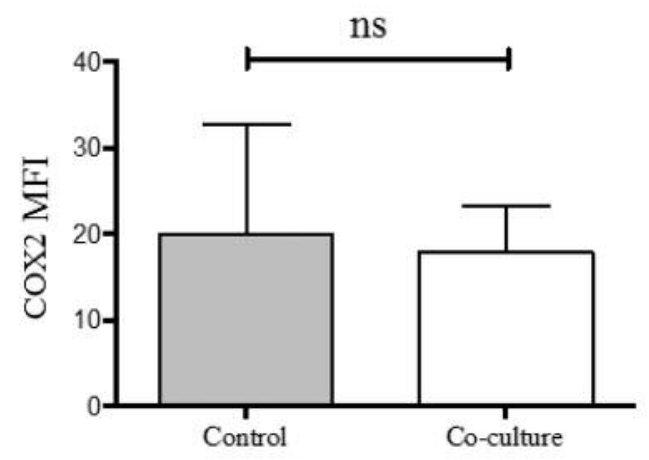

Figure 1. Effects of co-culture of canine peripheral blood mononuclear cells (PBMCs) with Shlb canine cancer cells on intracellular cyclooxygenase-2 (COX2) expression. A: Graphs show the overlay of flow cytometric expression of intracellular COX2 by Sh1b mammary cancer cells alone (gray filled histogram) and in co-culture with canine PBMCs (black solid line). Sh1b mammary cancer cells co-cultured with canine PBMCs showed a trend for overexpression of COX2 in comparison with Sh1b cultured alone. Co-culture of Sh1b mammary cancer cells and canine PBMCs reduced the expression of intracellular COX2 by the PBMCs $\left({ }^{* * *} p=0.0002\right)(B)$, specifically in the $C D 68^{+} C D 3^{-} P B M C$ cell fraction $(* p=0.0215)$ $(C)$, but did not induce alteration in COX2 expression in CD68-CD3+ cells $(D)$. Graphs show the mean $\pm S D$ of five independent experiments. pValues were calculated by two-tail paired Student t-test: $n s$, non significant.

incubated with primary antibody against COX2 (SP21, 1:50 dilution; Thermo Scientific, Lab Vision, Fremont, CA, USA) for $30 \mathrm{~min}$ at $4^{\circ} \mathrm{C}$. Cells were then washed in permeabilization buffer and incubated for $30 \mathrm{~min}$ at $4^{\circ} \mathrm{C}$ in the dark with secondary antibody for COX2 (Goat anti-rabbit IgG, Alexa Fluor 488, $2 \mu \mathrm{g} / \mathrm{ml}$; Life Technologies, Waltham, MA, USA). Finally, cells were washed with permeabilization buffer three times, re-suspended in FACS buffer and analyzed by flow cytometry. An appropriate isotype control was used to adjust for background fluorescence, and results are reported as the percentage expression or as the geometric mean fluorescence intensity (MFI). Flow cytometric analysis was performed using the data analysis software FlowJo version X (FlowJo LLC, Ashland, OR, USA).
Statistical analysis. Statistical Package for the Social Sciences version 19.0 (IBM Corp., Armonk, NY, USA) was used for statistical analysis. Comparisons among groups were performed with ANOVA or Student's $t$-test. Only differences with values of $p<0.05$ were considered significant.

\section{Results}

Effects on COX2 expression of co-culture of Shlb canine cancer cells and PBMCs from canine patients with tumor. To determine whether COX2 expression in canine mammary carcinoma cells was co-regulated by immune cells, we performed an in vitro co- 
A

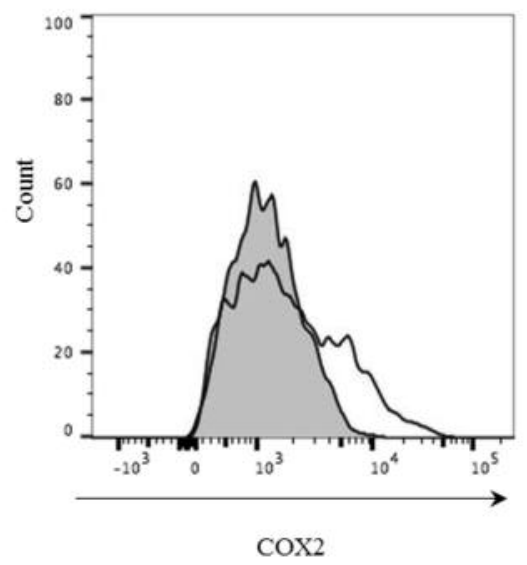

B

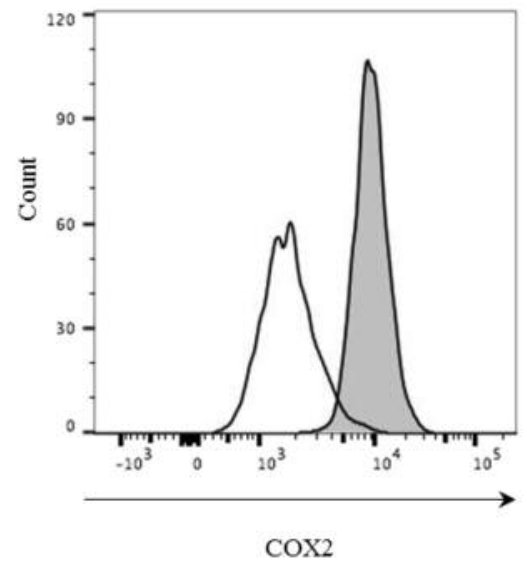

$\mathrm{C}$

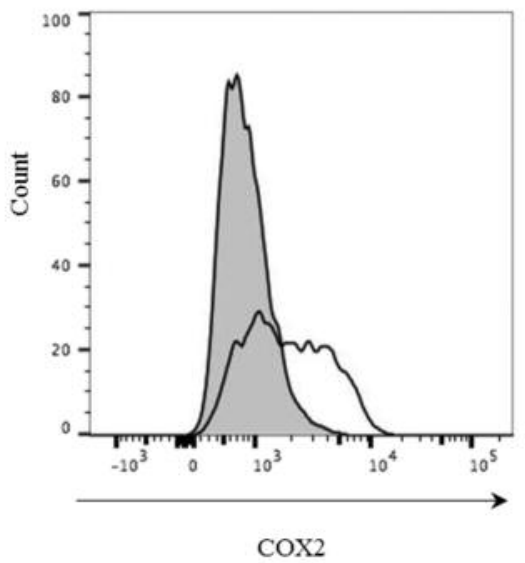

Figure 2. The effects of co-culture of THP1 human macrophage cell line and canine or human cancer cell lines on cyclo-oxygenase-2 (COX2) expression by the cancer cells. Co-culture of differentiated THP1 cells with Sh1b canine mammary cancer cells resulted in overexpression of intracellular COX2 (A), while that with BT474 human mammary cancer cells reduced intracellular COX2 production (B), but that with HT29 human colon cancer cells resulted in COX2 overexpression (C). Graphs show the overlay of flow cytometric expression of intracellular COX2 by cancer cells alone (gray filled histogram) and in co-culture (black solid line).

culture experiment. Sh1b mammary cancer cells co-cultured with canine PBMCs showed a trend for overexpression of COX2 in comparison with Sh1b cells cultured alone (Figure 1A). The expression of COX2 by PBMCs was significantly attenuated by their co-culture with the Sh1b cancer cell line $\left(p=0.0002\right.$, Figure 1B). Interestingly, CD68 ${ }^{+}$cells, putatively macrophages, are characterized by the highest expression of COX2 among PBMCs. The co-culture of Sh1b and canine PBMCs induced a decrease in expression of intracellular COX2 by the $\mathrm{CD}^{+} 8^{+}$fraction of canine PBMCs $(p=0.0215$, Figure $1 C)$, but did not induce significant alteration in intracellular $\mathrm{COX} 2$ expression by $\mathrm{CD}^{+}$PBMCs (Figure 1D).

Effects on COX2 expression of co-culture of cancer cell lines and human macrophages $d T H P 1$. The results obtained with PBMCs were validated in the $\mathrm{CD}^{+} 8^{+}$monocytic cell line THP1. Co-culturing dTHP1 cells with Sh1b cells indeed prompted up-regulation of COX2 in the canine mammary cancer cell line (Figure 2A).

Contrary to our expectations, co-culture of dTHP1 cells prompted a decrease in intracellular production of COX2 in BT474 human mammary cancer cells (Figure 2B).

In accordance with the results obtained with Sh1b cells, co-culture between HT29 cells and dTHP1 cells induced an increase of COX2 expression in the human colon cancer cells (Figure 2C).

Effects of cancer cell culture supernatants on intracellular COX2 expression by dTHP1 cells. In the co-culture model used above, immune cells were not allowed to make direct contact with the tumor cells. Thus to demonstrate the presence of soluble factors that might regulate intracellular COX2 expression in monocytic dTHP1 cells, cells were treated with conditioned medium recovered from $\mathrm{Sh} 1 \mathrm{~b}$ and HT29 cells cultured for $48 \mathrm{~h}$. dTHP1 cells treated with both Sh1b and HT29 culture supernatants showed a significant decrease of intracellular COX2 expression (Figure 3).

\section{Discussion}

In human mammary tumors, COX2 has crucial effects on the regulation and activity of T-cells and macrophages (19, 20, 25-27), contributing to tumor evasion of immune surveillance and supporting tumor development and metastasis $(11,19)$. Additionally in murine breast cancer models, selective COX2 inhibitors were shown to restrain breast cancer through augmentation of Th1 antitumor immune responses $(26,28)$. In canine mammary cancer, however, the association between COX2 and immune cells has so far only been described by immunohistochemistry, hence in a descriptive manner $(23,24)$. In order to determine the potential mutual regulation of $\mathrm{COX} 2$ between immune cells and mammary cancer cells, this aspect of the tumor microenvironment was mimicked in this study in vitro through the use of a transwell system. When in a first step the Sh1b canine mammary carcinoma cell line was cocultured with canine PBMCs from tumor patients, a trend for overexpression of COX2 was observed in comparison with Sh1b cells cultured alone. In turn, the expression of COX2 by PBMCs was significantly down-regulated. Almost all 


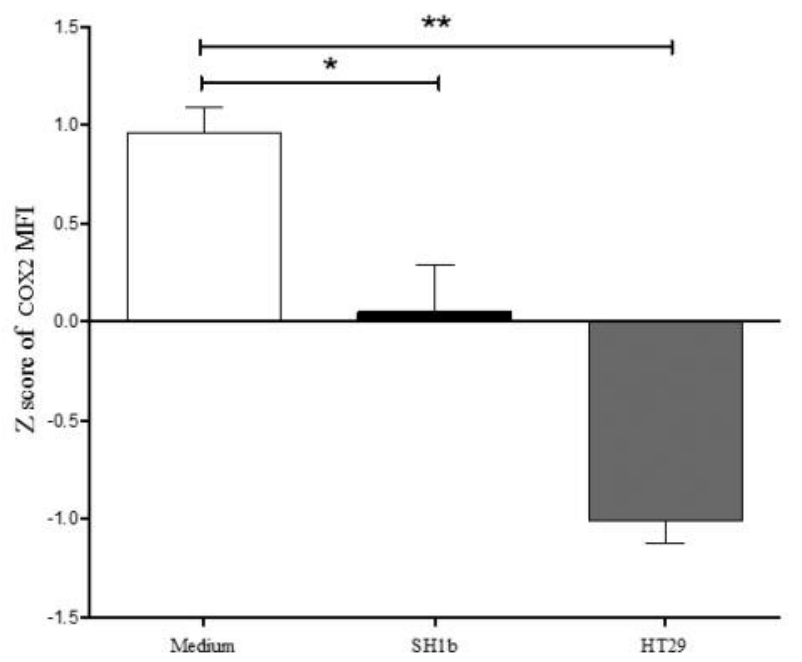

Figure 3. Effects of soluble factors derived from cultured cancer cells on intracellular cyclo-oxygenase-2 (COX2) expression by macrophages. Differentiated THP1 macrophages were cultured in medium alone or treated with $50 \% \mathrm{v} / \mathrm{v}$ conditioned medium from cultured Shlb canine mammary cancer cells or HT29 human colon cancer cells) for $48 \mathrm{~h}$ Graph shows the mean $\pm S D$-score of intracellular COX2 mean fluorescence intensity (MFI) of two independent experiments. p-Values were calculated by one-way ANOVA with Bonferroni's multiple comparisons post test: $* p=0.0257$ and $* * p=0.0028$.

COX2 expression was be ascribed to $\mathrm{CD}^{+} 8^{+} \mathrm{PBMCs}$, likely representing macrophages. Importantly, the co-culture did not induce considerable alteration in intracellular COX2 expression by $\mathrm{CD}^{+}$PBMCs.

Clinical and experimental human studies indicate that not only tumor-associated T-lymphocytes but also macrophage polarization are modulated by the tumor microenvironment in mammary cancer. In the tumor microenvironment, macrophages are 'educated', adopting a role that facilitates angiogenesis, matrix breakdown and tumor-cell motility in a COX2-dependent manner $(5,6,20,26,29)$.

Our findings are in accordance with the results of a study using co-culture between HCC1954 human mammary cancer cell line and mouse peritoneal macrophages, which showed increased COX2 expression in the cancer cells and elevated PGE2 level in the conditioned medium. Similar results were observed when human monocyte-derived macrophages were co-cultured with HCC1954 cells (27). More recently, another study using tumor-associated macrophages (TAMs) isolated from tumor tissues demonstrated that $\mathrm{COX}^{+}$TAMs promoted human mammary cancer cell proliferation and survival (22). Furthermore, in the same study COX2 in TAMs induced the expression of COX2 in breast cancer cells, which in turn promoted polarization of macrophages towards M2, hence, a positive-feedback loop exists between macrophages and breast cancer cells (22).
Although in dogs recent studies showed the relevance of a relationship between canine mammary cancer cells and macrophages that promotes macrophage polarization and cancer cell migration and invasion $(6,30)$, to our knowledge there are no studies on their potential interdependence in terms of COX2 regulation.

In order to validate the results obtained with the $\mathrm{CD} 68^{+}$ cell fraction of canine PBMCs, we next used the human $\mathrm{CD}^{+} 8^{+}$cell line THP1. The biological behavior of these cells is similar to that of monocyte-derived macrophages regarding adherence and phagocytic capacity, surface marker and cytokine expression (31), and they are widely used as monocyte/macrophage model $(22,31,32)$. Previously, the co-culture of THP1 and HCC1937 human mammary cancer cell line led to increased COX2 expression in cancer cells (22). In accordance with this, our results demonstrated that co-culture with dTHP1 prompted intracellular production of COX2 both in Sh1b canine mammary cancer cells and HT29 human colon cancer cells, but unexpectedly led to a decrease of COX2 in BT474 human mammary cancer cells. The effects were independent of cellular contact, but were transmitted by soluble mediators, from three different canine and human cancer cell lines to the monocytic cell line THP1.

We would like to emphasize that THP1 is a model cell line only. Therefore, we find it important that the results from this model mirrored the results from the $\mathrm{CD}^{+} 8^{+} \mathrm{PBMCs}$ from canine cancer patients. Moreover, other researchers demonstrated that COX2 in TAMs induced the expression of COX2 in cancer cells $(20,21)$. Importantly, this in turn, promoted macrophage polarization to the M2 tumor-supportive phenotype (27).

Our data support the notion that COX2-mediated immunomodulation in human breast cancer is a phenomenon shared by cancer cells and macrophages. This emphasizes the importance of exploring the tumor microenvironment as a whole $(1,22)$. The findings of the present study suggest for the first time that similarly to human breast cancer, COX2 regulation in tumor cells and macrophages in canine mammary cancer are interconnected and dependent on as yet unidentified soluble molecules.

\section{Conclusion}

Our data highlight the bi-directional regulation of COX2 in the canine mammary cancer microenvironment, implicating the contribution of cancer cells and macrophages. Immune cell-mediated induction of COX2 in canine mammary cancer cells may provide a mechanism whereby tumor-associated inflammatory cells contribute to tumor progression. COX2 regulation between cancer cells and macrophages in canine mammary cancer contributes to shaping a tolerogenic tumor microenvironment, and understanding this phenomenon might bring to light new potential therapeutic targets in canine mammary tumors. 


\section{Conflicts of Interest}

EJJ is a shareholder in Biomedical International R+D GmbH, Vienna, Austria. The Authors declare no further conflict of interest in regard to this study.

\section{Ethical Approval}

All applicable international, national, and institutional guidelines for the care and use of animals were followed.

\section{Acknowledgements}

The Authors would like to express their gratitude to all members of the Jensen-Jarolim laboratory for productive discussions and their support. This work was supported in part by grant F4606-B28 of the Austrian Science Fund FWF. Moreover, this study was also partially supported by Research Projects UID/AGR/04033/2013 and UID/MULTI/00211/2013, and by a Ph.D. scholarship SFRH/BD/78771/2011, all financed by the Portuguese Foundation for Science and Technology (FCT).

\section{References}

1 Hanahan D and Weinberg RA: Hallmarks of cancer: the next generation. Cell 144: 646-674, 2011.

2 Carvalho MI, Pires I, Prada J and Queiroga FL: A role for Tlymphocytes in human breast cancer and in canine mammary tumors. Biomed Res Int 2014: 130894, 2014.

3 Obeid E, Nanda R, Fu YX and Olopade OI: The role of tumorassociated macrophages in breast cancer progression (review). Int J Oncol 43: 5-12, 2013.

4 Carvalho MI, Pires I, Prada J and Queiroga FL: T-lymphocytic infiltrate in canine mammary tumours: clinic and prognostic implications. In Vivo 25: 963-969, 2011.

5 Raposo TP, Pires I, Carvalho MI, Prada J, Argyle DJ and Queiroga FL: Tumour-associated macrophages are associated with vascular endothelial growth factor expression in canine mammary tumours. Vet Comp Oncol 13(4): 464-474, 2013.

6 Beirao BC, Raposo T, Pang LY and Argyle DJ: Canine mammary cancer cells direct macrophages toward an intermediate activation state between M1/M2. BMC Vet Res 11: 151, 2015.

7 Dore M: Cyclo-oxygenase-2 expression in animal cancers. Vet Pathol 48: 254-265, 2011.

8 Queiroga FL, Pires I, Lobo L and Lopes CS: The role of Cox2 expression in the prognosis of dogs with malignant mammary tumours. Res Vet Sci 88: 441-445, 2010.

9 Park BW, Park S, Park HS, Koo JS, Yang WI, Lee JS, Hwang $\mathrm{H}$, Kim SI and Lee KS: Cyclo-oxygenase-2 expression in proliferative $\mathrm{Ki}-67$-positive breast cancers is associated with poor outcomes. Breast Cancer Res Treat 133: 741-751, 2012.

10 Millanta F, Asproni P, Canale A, Citi S and Poli A: COX2, mPGES-1 and EP2 receptor immunohistochemical expression in canine and feline malignant mammary tumours. Vet Comp Oncol 14(3): 270-280, 2014.

11 Greenhough A, Smartt HJ, Moore AE, Roberts HR, Williams AC, Paraskeva C and Kaidi A: The COX2/PGE2 pathway: key roles in the hallmarks of cancer and adaptation to the tumour microenvironment. Carcinogenesis 30: 377-386, 2009.
12 Hoellen F, Kelling K, Dittmer C, Diedrich K, Friedrich M and Thill M: Impact of cyclooxygenase-2 in breast cancer. Anticancer Res 31: 4359-4367, 2011.

13 Raposo T, Gregorio H, Pires I, Prada J and Queiroga FL: Prognostic value of tumour-associated macrophages in canine mammary tumours. Vet Comp Oncol 12: 10-19, 2014.

14 Murri AM, Hilmy M, Bell J, Wilson C, McNicol AM, Lannigan A, Doughty JC and McMillan DC: The relationship between the systemic inflammatory response, tumour proliferative activity, T-lymphocytic and macrophage infiltration, microvessel density and survival in patients with primary operable breast cancer. $\mathrm{Br}$ J Cancer 99: 1013-1019, 2008.

15 Martin F, Ladoire S, Mignot G, Apetoh L and Ghiringhelli F: Human FOXP3 and cancer. Oncogene 29: 4121-4129, 2010.

16 Merlo A, Casalini P, Carcangiu ML, Malventano C, Triulzi T, Menard S, Tagliabue E and Balsari A: FOXP3 expression and overall survival in breast cancer. J Clin Oncol 27: 1746-1752, 2009.

17 Strauss L, Bergmann C, Szczepanski M, Gooding W, Johnson JT and Whiteside TL: A unique subset of $\mathrm{CD} 4{ }^{+} \mathrm{CD} 25^{\text {highFOXP3 }}{ }^{+} \mathrm{T}-$ cells secreting interleukin-10 and transforming growth factorbeta1 mediates suppression in the tumor microenvironment. Clin Cancer Res 13: 4345-4354, 2007.

18 Strauss L, Bergmann C and Whiteside TL: Human circulating $\mathrm{CD} 4{ }^{+} \mathrm{CD} 25^{\text {highFOXP }}{ }^{+}$regulatory T-cells kill autologous $\mathrm{CD}^{+}$ but not $\mathrm{CD}^{+}{ }^{+}$responder cells by Fas-mediated apoptosis. J Immunol 182: 1469-1480, 2009.

19 Markosyan N, Chen EP, Evans RA, Ndong V, Vonderheide RH and Smyth EM: Mammary carcinoma cell derived cyclooxygenase 2 suppresses tumor immune surveillance by enhancing intratumoral immune checkpoint activity. Breast Cancer Res 15(5): R75, 2013.

20 Eruslanov E, Daurkin I, Ortiz J, Vieweg J and Kusmartsev S: Pivotal Advance: Tumor-mediated induction of myeloid-derived suppressor cells and M2-polarized macrophages by altering intracellular PGE(2) catabolism in myeloid cells. J Leukoc Biol 88: 839-848, 2010.

21 Stewart DA, Yang Y, Makowski L and Troester MA: Basal-like breast cancer cells induce phenotypic and genomic changes in macrophages. Mol Cancer Res 10(6): 727-738, 2012.

22 Hou Z, Falcone DJ, Subbaramaiah K and Dannenberg AJ: Macrophages induce COX2 expression in breast cancer cells: role of IL-1beta autoamplification. Carcinogenesis 32: 695-702, 2011.

23 Carvalho MI, Pires I, Prada J, Ferreira AF and Queiroga FL: Positive Interplay Between CD3+ T-lymphocytes and Concurrent COX2/EGFR expression in canine malignant mammary tumors. Anticancer Res 35: 2915-2920, 2015.

24 Carvalho MI, Pires I, Prada J, Raposo TP, Gregorio H, Lobo L and Queiroga FL: High COX2 expression is associated with increased angiogenesis, proliferation and tumoural inflammatory infiltrate in canine malignant mammary tumours: a multivariate survival study. Vet Comp Oncol 15(2): 619-631, 2016.

25 Pockaj BA, Basu GD, Pathangey LB, Gray RJ, Hernandez JL, Gendler SJ and Mukherjee P: Reduced T-cell and dendritic cell function is related to cyclo-oxygenase- 2 overexpression and prostaglandin $\mathrm{E}_{2}$ secretion in patients with breast cancer. Ann Surg Oncol 11: 328-339, 2004.

$26 \mathrm{Na}$ YR, Yoon YN, Son DI and Seok SH: Cyclooxygenase-2 inhibition blocks M2 macrophage differentiation and suppresses metastasis in murine breast cancer model. PLoS One 8(5): e63451, 2013. 
27 Li H, Yang B, Huang J, Lin Y, Xiang T, Wan J, Li H, Chouaib $\mathrm{S}$ and Ren G: Cyclooxygenase-2 in tumor-associated macrophages promotes breast cancer cell survival by triggering a positive-feedback loop between macrophages and cancer cells. Oncotarget 6: 29637-29650, 2015.

28 Markosyan N, Chen EP, Ndong VN, Yao Y, Sterner CJ, Chodosh LA, Lawson JA, Fitzgerald GA and Smyth EM: Deletion of cyclo-oxygenase 2 in mouse mammary epithelial cells delays breast cancer onset through augmentation of type 1 immune responses in tumors. Carcinogenesis 32: 1441-1449, 2011.

29 Kadioglu E and De Palma M: Cancer metastasis: perivascular macrophages under watch. Cancer Discov 5: 906-908, 2015.

30 Krol M, Pawlowski KM, Majchrzak K, Gajewska M, Majewska A and Motyl T: Global gene expression profiles of canine macrophages and canine mammary cancer cells grown as a coculture in vitro. BMC Vet Res 8: 16, 2012.
31 Daigneault M, Preston JA, Marriott HM, Whyte MK and Dockrell DH: The identification of markers of macrophage differentiation in PMA-stimulated cells and monocyte-derived macrophages. PLoS One 5(1): e8668, 2010.

$32 \mathrm{Wu}$ TH, Li YY, Wu TL, Chang JW, Chou WC, Hsieh LL, Chen JR and Yeh KY: Culture supernatants of different colon cancer cell lines induce specific phenotype switching and functional alteration of THP1 cells. Cell Immunol 290: 107-115, 2014.

Received February 3, 2018

Revised March 12, 2018

Accepted March 14, 2018 